\title{
Idiopathic spontaneous perforation of the upper urinary tract. A presentation of 4 cases
}

\author{
Ioannis Katafigiotis ${ }^{1}$, Ioannis Adamakis ${ }^{2}$, Alexandra Zormpala ${ }^{3}$, Christos Pournaras ${ }^{1}$, \\ Konstantinos Stravodimos ${ }^{2}$ \\ ${ }^{1}$ Resident in Urology, University of Athens Medical School, Athens, Greece; \\ ${ }^{2}$ Assistant Professor in Urology, University of Athens Medical School, Athens, Greece; \\ ${ }^{3}$ Radiologist, Radiology Department, Laikon General Hospital, Athens, Greece.
}

\begin{abstract}
Summary Spontaneous perforation of the collecting system constitutes a rare entity masked by the presentation of a typical renal colic. However, it should not be forgotten when managing patients with colic, since missed diagnoses may carry significant morbidity. We herein present a series of spontaneous perforation of the collecting system without an apparent obstruction site evident in helical CT urography. Four consecutive patients who presented with typical renal colic were initially subjected to KUB and renal ultrasound imaging and were ultimately diagnosed with perforation of the collecting system via contrast enhanced- helical CT urography. Despite thorough evaluation, the cause responsible obstructive was not discovered and an exclusion diagnosis of idiopathic collecting system perforation was assigned to all patients. Due to the rarity of a spontaneous perforation traditional retrograde urography was performed in an effort to identify the possible cause but was also unrevealing. All patients were successfully treated with endourological means. Perforation of the collecting system without an evident obstructive cause is a rare entity with obscure etiology. A reasonable but yet unconfirmed speculation is that of a transient obstruction capable of a significant increase of intraluminal pressures.
\end{abstract}

KEY WORDS: Spontaneous ureter rupture; Idiopathic upper urinary tract perforation.

Submitted 28 April 2014; Accepted 31 May 2014

\section{INTRODUCTION}

Spontaneous perforation of the collecting system is a rather rare disease entity with relevant reports dating back to the 60s. Several latter reports presented a variety of cases each within an individual clinical setting. In fact, spontaneous perforation of the ureter has been encountered as a complication of cystectomy with orthotopic ileal neobladder formation (1) or lupus vasculitis (2). Even though these reports have termed respective cases of upper tract perforation as spontaneous, it is reasonable to assume that ureteral rupture with impaction of a ureteral calculus as the triggering event would represent the most common scenario. Interestingly, certain cases with no obvious cause (excluding even calculus disease) have also been reported (3).

Helical computed tomography (CT) urography is currently considered the standard of practice in the evaluation of an episode of renal colic. Delayed scans are already reported as the optimum for the diagnosis of traumatic ureteral rupture and respective urine leaks (4). Even though no data have been published on their value in diagnosis of spontaneous upper tract rupture, it seems reasonable to apply the technique in this setting.

After thorough evaluation with the above mentioned techniques in 4 cases of persistent renal colic with confirmed urinoma formation an obstructive cause was not revealed. Therefore a diagnosis of spontaneous rupture of the upper tract was set and respective data and comments are herein presented.

\section{Materials AND MEthods}

During a 14 month period, from May 2011 to July 2012, 4 patients ( 2 male, 2 female, mean age 71.5 years, age range 67-79 years) presented with signs and symptoms of acute renal colic in 3 different urological departments (2 to an urban university referral centre-University of Athens, Athens, Greece and 2 to rural county-affiliated hospitals). Patients underwent the conventional diagnostic work-up routinely performed in such cases including blood and urine tests (urinalysis with urine culture, blood cell counts and biochemical panel).

\section{Imaging studies}

All patients underwent renal ultrasonography and kidneys, ureters and bladder (KUB) X-ray. In all patients abdominal helical CT urography was performed using a helical scanner from the level of the kidneys to the pubic symphysis, in breath-hold status, with a slice thickness of $5 \mathrm{~mm}$ and a pitch of I.5. Intravenous non-ionic contrast was administered at a rate of $3 \mathrm{ml}$ per second for a 
total volume of 100-130 ml. The CT scans were obtained as part of a CT renal protocol that included nonenhanced imaging and imaging in both nephrographic and delayed excretory phases. Delayed excretory phase scans of the urinary tract were acquired 10-15 minutes after the injection. In order to obtain a more accurate view of the urinary tract and increase the sensitivity of the work-up, retrograde urography was also used. The latter was used after the patient had undergone evaluation with delayed CT scan, so the conclusions based on CT imaging were minimally biased.

\section{RESULTS}

Ultrasound findings raised the suspicion for urinary tract continuity disruption with perinephric fluid collection of mixed echogenic characteristics in all cases.

Delayed scans of helical CT-urography set the diagnosis of upper tract disruption in all four patients. Contrast medium leakage in the periureteral and perirenal space was the main finding (Figures 1, 2).

Retrograde pyelography (performed during stent insertion) confirmed the point of extravasation at the ureteropelvic junction in three patients and the upper third of the ureter in the remaining. An obvious obstructive cause was not demonstrated in either case.

All patients were then managed by endourological approaches using a double J stent under fluoroscopic guidance.

Drainage of the urinoma was eventually required in one patient who developed septic complications rendering the case an absolute emergency.

Discussion and Full list of References are posted in Supplementary Materials on www.aiua.it

\section{REFERENCES}

1. Deliveliotis Ch, Chrisofos M, Argyropoulos V, et al. Spontaneous rupture of the ureter after cystectomy and creation of orthotopic ileal neobladder: treatment with percutaneous nephrostomy and drainage. J Endourol. 2003; 17:33-5.

2. Benson CH, Pennebaker JB, Harisdangkul V, Songcharoen S. Spontaneous ureteral rupture in a patient with systemic lupus erythematosus. South Med J. 1983; 76:1053-5.

3. Ertugrul A, Yücel S, Ilker Y, Akdas A. Use of double J stent in a case of spontaneous ureteral extravasation of urine. Arch Esp Urol. $2000 ; 53: 491-3$

Figures 1, 2.

Delayed excretory CT scans demonstrating contrast medium leakage in the perirenal and periureteral space compatible with urinary tract disruption.
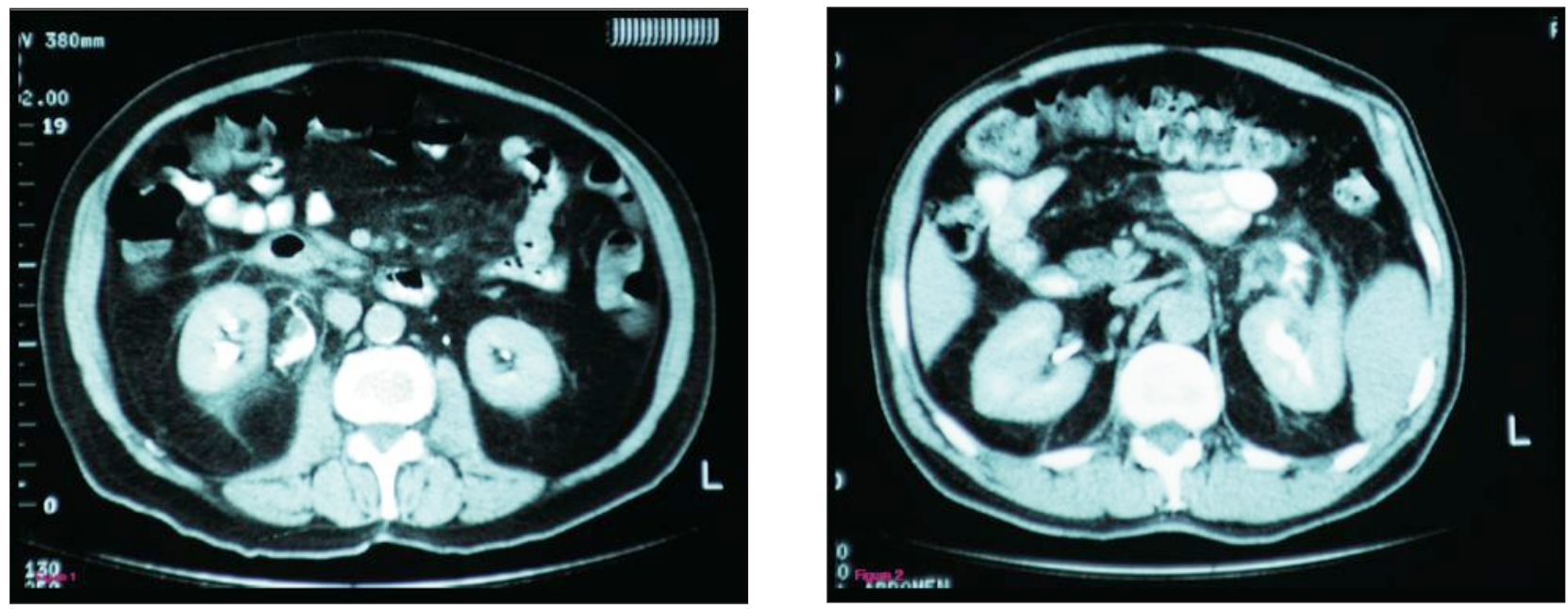

\section{Correspondence}

Ioannis Katafigiotis, MD (Corresponding Author)

katafigiotis@yahoo.com

Christos Pournaras, MD

christpourn@gmail.com

Ioannis Adamakis, MD

yianton@hotmail.com

Konstantinos Stravodimos, MD

kgstravod@yahoo.com

Alexandra Zormpala, MD

azormpala@med.uoa.gr

Department of Urology, Athens University Medical School - LAIKO

Hospital, 17 Agiou Thoma str., 11527 Athens, Greece 